\title{
Relationship between nasopharyngeal and bronchoalveolar microbial communities in clinically healthy feedlot cattle
}

Mohamed M. Zeineldin', James F. Lowe', Elsbeth D. Grimmer ${ }^{1}$, Maria R. C. de Godoy², Mohamed M. Ghanem³, Yassein M. Abd El-Raof ${ }^{3}$ and Brian M. Aldridge ${ }^{1 *}$

\begin{abstract}
Background: The importance of upper airway structure in the susceptibility of the lower respiratory tract to colonization with potential pathogens is well established. With the advent of rapid, high throughput, next generation sequencing, there is a growing appreciation of the importance of commensal microbial populations in maintaining mucosal health, and a realization that bacteria colonize anatomical locations that were previously considered to be sterile. While upper respiratory tract microbial populations have been described, there are currently no published studies describing the normal microbial populations of the bovine lower respiratory tract. Consequently, we have little understanding of the relationship between upper and lower respiratory tract microbiota in healthy cattle. The primary objective of our study was to characterize the composition, structure and relationship of the lower and upper respiratory microbial communities in clinically healthy feedlot cattle. Nasopharyngeal swabs (NPS), and bronchoalveolar lavage (BAL) fluid, were collected from clinically healthy feedlot calves $(n=8)$. Genomic DNA from each sample was extracted, and the V3-V4 hypervariable region of the bacterial $16 \mathrm{~S}$ rRNA gene was amplified and sequenced using Illumina Miseq platform.
\end{abstract}

Results: Across all samples, the most predominant phyla were Proteobacteria, Actinobacteria and Firmicutes. The most common genera were Rathayibacter, Mycoplasma, Bibersteinia and Conynebacterium. The microbial community structure was distinct between these two biogeographical sites. Most of the bacterial genera identified in the BAL samples were also present in the NPS, but biogeographical-specific genera were enriched in both the NPS (Rathayibacter) and BAL (Bibersteinia) samples. There were strong associations between the presence of certain taxa at each specific location, and strong correlations between the presence of specific taxa in both the NPS and BAL samples.

Conclusions: The correlation between the presence of specific taxa in both the NPS and BAL samples, supports the notion of a mutualistic interrelationship between these microbial communities. Future studies, in large cohorts of animals, are needed to determine the role and clinical importance of the relationships of respiratory tract microbial communities with health, productivity, and susceptibility to the development of respiratory disease, in growing cattle.

Keywords: Feedlot, Microbiota,16S rRNA gene, Next generation sequencing, Respiratory tract

\footnotetext{
* Correspondence: ba311@illinois.edu

${ }^{1}$ Integrated Food Animal Management Systems, Department of Veterinary

Clinical Medicine, College of Veterinary Medicine, University of Illinois at

Urbana-Champaign, 241 LAC, 1008 W Hazelwood Dr, Urbana, IL 61802, USA

Full list of author information is available at the end of the article
} 


\section{Background}

Bovine respiratory disease (BRD) is one of the most commonly reported diseases in cattle, especially in intensely raised, recently weaned and newly transported feedlot cattle [1]. The etiopathogenesis of BRD is complex, and results from the complex interaction of bacterial and viral pathogens under the influence of a wide range of host and environmental risk factors [2]. Despite many advancements in management and therapeutics, BRD and its sequelae, continue to be the leading causes of animal morbidity, mortality, welfare concern and production loss to the industry [3]. The importance of the mucosal microbial community structure in maintaining epithelial health and homeostasis of the skin, gastrointestinal and reproductive tracts has been recognized for many years, but its significance has only relatively recently been demonstrated in the respiratory tract [4]. Despite the implication of resident bacterial populations in the etiopathogenesis of BRD [5], the detailed structure of the lower airway microbiota has not been investigated. The characterization of airway microbiota is expected to provide insights into the pathophysiology of $\mathrm{BRD}$, and open the door for new health management strategies. Historically, characterization of the cattle microbiota has relied heavily on culture-dependent techniques which have mainly focused on the identification of major pathogens that can be easily cultured. While useful, this approach is unable to provide information on those organisms that cannot be easily cultured, but are likely present at these sites, and so only provides a narrow understanding of the complexity of these clinically important microbial ecosystems [6]. Advances in next generation sequencing and bioinformatics have facilitated the characterization of the composition and diversity of complex microbial populations at mucosal sites, and have provided remarkable insight into the interaction of these populations with the host [7]. Most next generation sequencing studies in cattle have focused on the gastrointestinal tract $[8,9]$, but the existence of important resident microbial populations in the nasopharynx has also been described [10]. The microbiota of the respiratory tract is of particular interest due to its association with BRD [5]. It is widely recognized that the upper respiratory tract microbiome provides a first line of defense against foreign invaders through competition, and interaction, with potential mucosal pathogens [11]. While the composition and development of the nasopharyngeal microbiota in feedlot cattle has been recently described $[5,10,12,13]$, there are no published studies describing lower respiratory tract microbial communities in feedlot cattle. Interestingly, until recently the lower respiratory tract in healthy humans has traditionally been considered as sterile using culture-dependent or conventional molecular techniques $[14,15]$. Technical advances in culture-independent techniques have shown that the respiratory tract of healthy people is not sterile, but is composed of a complex and previously unappreciated microbial community $[16,17]$, and that certain configurations of the microbiota may be associated with development of respiratory disease [18]. In view of the importance of respiratory disease to the industry, our objectives were to characterize the composition and structure of the upper and lower respiratory microbiota, and to determine the relationship between the nasopharyngeal and bronchoalveolar microbiotas in clinically healthy feedlot cattle.

\section{Methods}

\section{Animal populations and sample collection}

A total of eight, six to eight month old, single-source, Charolais feedlot calves (mean body weight $348.47 \pm 14.59 \mathrm{~kg}$ ), were enrolled in our study in April 2016, approximately 60 days after arrival at the South Farms-Beef Cattle and Sheep Field Laboratory, University of Illinois at UrbanaChampaign- USA. All calves were clinically healthy, with no history of receiving any antimicrobial drugs prior to, or after arrival at the feedlot. The calves were vaccinated immediately after arrival at the feedlot, with a modified live virus vaccine against IBR, BVDV (Types I and II), BPIV-3 and BRSV (5 ml IM; Bovi-Shield Gold FP5 L5 HB Cattle Vaccine, Zoetis Animal Health), and dewormed with a topical anthelminthic (Noromectin Pour-On Solution, Norbrook $^{\otimes}$ Inc. USA). The calves were fed a mixed ration (20\% silage, $20 \%$ modified wet distillers grains with soluble, $10 \%$ dry supplement, and 50\% high moisture corn), and given free access to water. The use of animals for this study was approved by the University of Illinois Institutional Animal Care and Use Committee (IACUC Protocol: \#15064) and all of the experimental protocols were performed in accordance with relevant guidelines and regulations set by IACUC.

Physical exams, including rectal temperature, respiratory rate and pulse rate, were performed with the calves standing in the hydraulic chute. A clinical lung score was recorded using the microphone of an automated Whisper stethoscope (Whisper, Geissler Corp, Plymouth, MN). Briefly, the stethoscope was placed over the 5th intercostal space of the right thoracic wall, approximately $10 \mathrm{~cm}$ above the elbow, at a site known to encompass the apical lung lobes. Recorded lung sounds were then automatically transmitted wirelessly to a computer located within $2 \mathrm{~m}$ of the stethoscope, and analyzed using software rendering a 5-point lung score scales [19]. The program software is designed to remove heart sounds and potential interference from the environment, and classifies acoustic patterns in to lung scores ranging from 1 to 5 ( 1 = normal, $2=$ mild acute, 3 = moderate acute, $4=$ severe acute, and 5 = chronic).

Feedlot calves with a rectal temperature $<39.4{ }^{\circ} \mathrm{C}$, respiratory rate $<50$ breaths $/ \mathrm{min}$, pulse rate $<120$ 
beats/min and lung score $\leq 2$ were defined as healthy, and selected for sampling.

Following physical examination, a single, deep NPS was collected from each calf, using a 33-in.-long double guarded PVC culture swab (Kalayjian Industries, Inc. U.S.A.) according to published techniques [20]. Briefly, the calf's nostrils were cleaned with a disposable wipe before collection. The NPS was carefully inserted into the ventral meatus of the nose, and advanced approximately $2 / 3$ of the dorsal head length. Once in place, the end of the swab was exposed to a length of approximately $1-2$ in., by withdrawing the outer sleeve, and the sampling unit was then firmly rotated through $360^{\circ}$ against the pharyngeal wall, for $20-30 \mathrm{~s}$. The cotton tipped swab was then retracted back in to the outer sheath, and the whole swab was removed gently from the animal's nose. Each cotton swab was then broken off into a sterile $2 \mathrm{ml}$ cryo-tube, transported on dry ice to the laboratory, and stored at $-20{ }^{\circ} \mathrm{C}$ pending further processing.

Following NPS sampling, the calves were sedated with Xylazine hydrochloride $(0.1 \mathrm{mg} / \mathrm{kg} \mathrm{IM})$ (Rompun ${ }^{\circ}$, Bayer health care LLC, Kansas) and the nostrils were cleaned with dry gauze. BAL was performed with the sedated calves in a standing position, using a sterilized, flexible BAL tube (BAL300 - $300 \mathrm{~cm}$ length, $10 \mathrm{~mm}$ outside diameter, $2.5 \mathrm{~mm}$ internal diameter, balloon volume $10 \mathrm{~cm}^{3}$, MILA International, Inc. U.S.A.) [21]. The BAL catheter was introduced into the nostril, directed into the ventral meatus, and then advanced until it encountered resistance in the caudal pharynx. At this point, the calf's head and neck were held straight, in maximal extension, to allow the catheter to pass into the trachea during the inspiratory phase of the respiratory cycle. On reaching a wedged position in the lower respiratory airway, the catheter was secured by inflating the balloon cuff with $5 \mathrm{~cm}^{3}$ of air. For sampling, $120 \mathrm{mls}$ of sterile saline was infused through the catheter lumen, using $60 \mathrm{ml}$ syringes attached to a stopcock and catheter tipped adapter. Immediately after the $120 \mathrm{ml}$ infusion, negative pressure was applied to aspirate airway fluid, which was immediately placed into a sterile $50 \mathrm{ml} \mathrm{speci-}$ men tube. The BAL samples were stored on ice until processing, approximately $2-4 \mathrm{~h}$ after collection. The collected BAL was centrifuged at $14,000 \times \mathrm{g}$ for $10 \mathrm{~min}$. The pellets were re-suspended in $500 \mu \mathrm{L}$ sterile PBS and stored at $-20{ }^{\circ} \mathrm{C}$ pending further analysis.

\section{Genomic DNA extraction}

Genomic DNA extraction was performed from each NPS and BAL sample using the PowerFecal ${ }^{\circ}$ DNA isolation Kit (MO BIO Laboratories, Inc., Carlsbad, CA, USA), according to the manufacturer's instructions. Briefly, each sample was transferred to a dry bead tube with $60 \mu \mathrm{l}$ of
Solution $\mathrm{C} 1$ and $750 \mu \mathrm{l}$ of Bead Solution, heated at $65{ }^{\circ} \mathrm{C}$ for $10 \mathrm{~min}$, and settled in Bullet Blender 24 Gold tube holder (Next Advance, Inc., Averill Park, NY, USA). The tubes were vortexed at maximum speed for $10 \mathrm{~min}$ to achieve microbial cell disruption. The PowerFecal ${ }^{\circ}$ DNA isolation Kit protocol was used to complete the extraction, according to manufacturer instructions. Purified DNA was eluted into $50 \mu \mathrm{l}$ of Solution C6 rather than $100 \mu \mathrm{l}$ to increase the DNA concentration. Total DNA concentration was quantified using a Nanodrop ${ }^{\text {ma }}$ spectrophotometer (NanoDrop Technologies, Rockland, DE, USA) at wavelengths of 260 and $280 \mathrm{~nm}$, and the integrity was confirmed by agarose gel electrophoresis.

\section{Fluidigm access Array amplification of the V3-V4 hypervariable region of $16 \mathrm{~S}$ rRNA genes and Illumina sequencing}

Genomic DNA was subject to Fluidigm Access Array Amplification (Fluidigm Corporation, South San Francisco, CA, USA). Prior to amplification, all DNA samples were measured on a Qubit ${ }^{\mathrm{Tm}}$ fluorometer (Life technologies, Grand Island, NY, USA) using the High Sensitivity DNA Kit (Agilent Technologies, Santa Clara, CA, USA) [22].

Briefly, the primer sequences F357-for (CCTACGGGA GGCAGCAG) and R806-rev (GGACTACNVGGGTWT CTAAT) were used to amplify the V3-V4 hypervariable region of the $16 \mathrm{~s}$ rRNA gene. PCR reactions were performed on a Fluidigm Biomark $\mathrm{HD}^{\mathrm{ma}}$ PCR machine (Fluidigm Corporation, South San Francisco, CA, USA) using the default Access Array cycling program without imaging.

Harvested product was quantified on a Qubit ${ }^{\mathrm{m}}$ fluorometer (Life technologies, Grand Island, NY, USA) and stored at $-20{ }^{\circ} \mathrm{C}$. All samples were run on a Fragment Analyzer $^{\mathrm{mm}}$ (Advanced Analytics, Ames, IA, USA), and the amplicon regions were quantified. PCR products were then size selected on a $2 \%$ agarose $\mathrm{E}^{- \text {gel }^{\mathrm{Tm}}}$ (Life technologies, Grand Island, NY, USA) and extracted from the isolated gel slice with the QIAquick Gel extraction kit (Qiagen, Valencia, CA, USA). Cleaned, size-selected product was examined on an Agilent Bioanalyzer ${ }^{\text {rm }}$ to confirm appropriate profile, and for the determination of average size. The final pooled Fluidigm libraries were transferred to the DNA Services lab at the W. M. Keck Center for Comparative and Functional Genomics (University of Illinois at Urbana-Champaign, Urbana, IL, USA) for Illumina sequencing. The Illumina MiSeq $^{\text {Tw }}$ platform (Illumina, San Diego, CA, USA) was used to sequence the V3- V4 region of the 16S rRNA gene according to the Illumina instructions.

\section{Sequence data processing and statistical analysis}

The raw sequence data were preprocessed and analyzed using the open-source software package, Quantitative 
Insights Into Microbial Ecology (QIIME ${ }^{\circledR}$ ) version 1.9 (http://qiime.org/) [23]. Sequences were filtered for quality the default parameters of the split_libraries.py command; minimum sequence length equal 200, maximum sequence length equal 1000, a Phred score of less than 25 , maximum number of ambiguous bases equal 6 and homopolymer runs of $>6$ bp [24]. Chimeric sequences were detected and removed using UCHIME [25]. The remaining sequences were clustered into operational taxonomic units (OTUs) using open reference OTU selection protocols (97\% identity cutoff) with the UCLUST algorithm [26], and assigned a taxonomic classification against the Greengenes ${ }^{\oplus}$ database [27].

The core microbiota, those microbes shared among all sampled calves, was identified at the genus level. For subsequent alpha and beta diversity analysis, the OTU table was randomly subsampled and rarefied to 2400 sequences per sample. Alpha diversity (an estimate of bacterial community richness in a sample) was calculated within QIIME $^{\bullet}$ using the Chao1 (an estimate of species richness), observed species (the total number of microbial species present in a community), and phylogenetic diversity (PD whole tree) (an estimate of the biodiversity which incorporates phylogenetic difference between species). Beta-diversity (an estimate of bacterial communities expression of diversity between different sites) was calculated using weighted and unweighted UniFrac distance [28] and clustering of the samples based on distance was visualized on principal coordinate analysis (PCoA) plots using EMPeror ${ }^{\circ}$ [29].

The OTU relative abundance values were analyzed using the linear discriminant analysis (LDA) effect size (LEfSe) algorithm, to identify OTUs that display significant differences between the two sites [30]. Additionally, a cladogram was produced using the online LEfSe tool. The algorithm first used the non-parametric factorial Kruskal-Wallis test to detect taxa with significantly different abundance, followed by pairwise Wilcoxon test to detect biological consistency between NPS and BAL samples, and then used LDA to estimate the effect size of each differentially abundant feature. Statistical analyses were performed using JMP ${ }^{\circ} 12.12$ (SAS Institute Inc., North Carolina, USA).

For characterization of NPS versus BAL microbiota, analysis of bacterial abundance between the NPS and BAL samples in healthy calves was performed using nonparametric Wilcoxon tests. Alpha diversity metrics (Chaol, observed species and PD whole tree) were also compared between the two groups using a non-parametric Wilcoxon tests in JMP 12.12 software. The weighted and unweighted UniFrac distances for NPS and BAL samples were also compared using nonparametric ANOSIM test (analysis of similarities) with 999 Monte Carlo permutations.
To further explore the possible relationships between upper and lower respiratory tract microbial communities, multivariate analysis (linear correlation matrixes) was performed using JMP $^{\circ} 12.12$ (SAS Institute Inc., North Carolina, USA). In view of the large number of comparisons, an additional, more conservative approach was adopted using multiple linear forward regression analysis. Forward linear regression analysis was performed using the SPSS version 22 (IBM Corp, Version 22.0, Armonk, NY, 2013) statistical package. Differences with a $P$ value $\leq 0.05$ were considered significant for all analyses.

Fastq data obtained in the current study were uploaded to the sequence read archive (SRA) on the National Center for Biotechnology Information (NCBI) website (http:// www.ncbi.nlm.nih.gov/sra), to make the files available for a public database with bio-project accession number PRJNA323521.

\section{Results}

\section{Sample population}

Eight, six to eight month old, clinically healthy feedlot cattle were enrolled into the study. There was no detectable evidence of clinical signs of BRD in any of the enrolled calves. The median (range) of rectal temperature $\left(\mathrm{C}^{\circ}\right)$, heart rate (beat $/ \mathrm{min}$ ), respiratory rate (breaths $/ \mathrm{min}$ ) and Whisper lung scores were 39.27 (38.55-39.38), 94 (64-118), 36 (26$48)$ and $1(1-2)$ respectively.

\section{Overall sequence analysis}

The sequencing analysis of the V3-V4 hypervariable regions of the bacterial 16S rRNA resulted in a total of 298,875 sequences in all NPS and BAL samples. The number of sequences per sample ranged from 2419.0 to 48,092.0 (mean 18,679.68, SD 13963.19) and comprised 195 OTUs (97\% identity cutoff) across all samples (Additional file 1: Table S1).

\section{Taxonomic characterization of the nasopharyngeal versus bronchoalveolar microbiota}

At the phylum level, the dominant bacterial phyla across all NPS and BAL samples were Proteobacteria (24.6\%), Actinobacteria (24.4\%), Firmicutes (15.8\%), Bacteroidetes (13.5\%) and Tenericutes (10.4\%). Overall, $4.2 \%$ of NPS and $14.6 \%$ of BAL sequences could not be classified at the phylum-level (Fig. 1). The relative abundance of each phylum between individuals was highly variable across all the NPS and BAL samples (Fig. 1).

In NPS samples, we observed a predominance of Actinobacteria (43.9\% versus $4.9 \%$ in BAL samples) and Tenericutes (12.1\% versus $8.8 \%$ in BAL samples). While in BAL samples we observed a predominance of Proteobacteria (33.3\% versus $15.9 \%$ in NPS samples), Bacteroidetes (18.2\% versus $8.8 \%$ in NPS samples) and Fusobacteria (3.4\% versus $0.1 \%$ in NPS samples). 

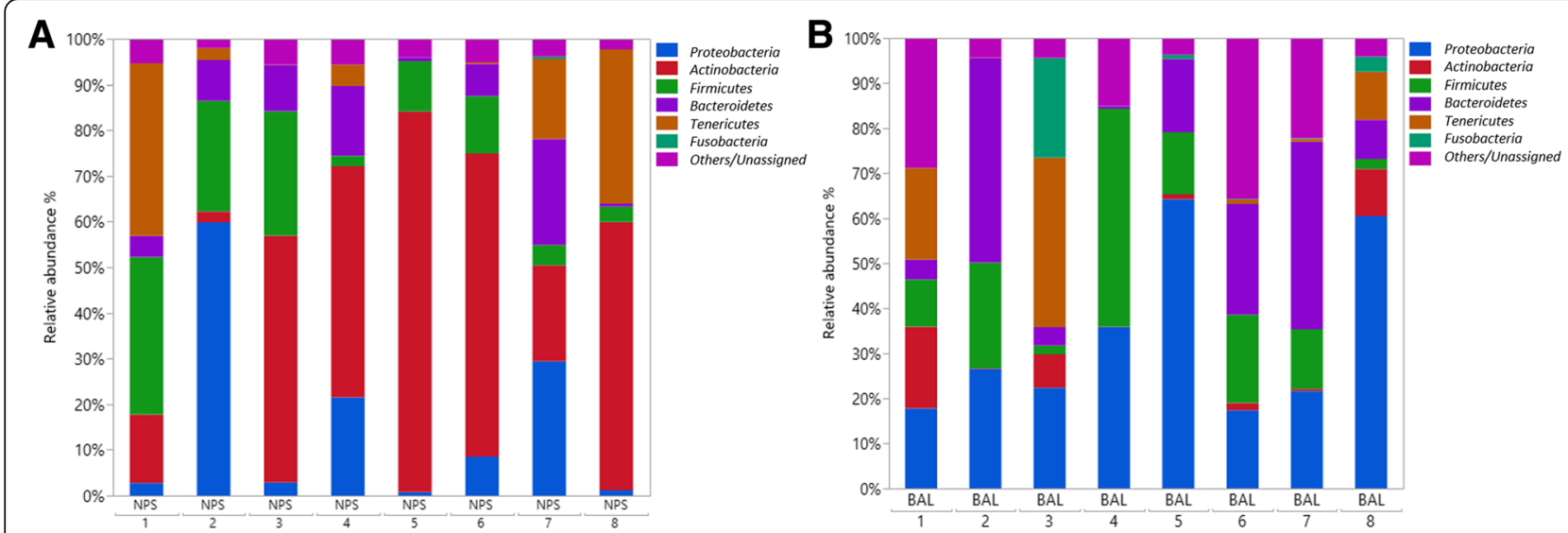

Fig. 1 Relative abundance of bacterial 16S rRNA gene sequences at the phylum level observed in the NPS $\mathbf{a}$ and BAL $\mathbf{b}$ samples from 8 healthy feedlot calves. Only those bacterial phyla that averaged more than $1 \%$ of the relative abundance across all samples when sequencing V3-V4 hypervariable regions are displayed. All other unassigned and classified OTUs belonged to phyla comprising less than $1 \%$ of the total abundance represented as others/Unassigned

At the genus level, there were 57 genus comprising greater than $0.1 \%$ of sequences in both NPS and BAL samples (Additional file 2: Table S2). The most abundant genera in the airway microbiota across all the NPS and BAL samples were Rathayibacter (12.24\%), followed by Mycoplasma (10.84\%), Bibersteinia (7.20\%), Corynebacterium (6.32\%), Prevotella (5.98\%), and Clostridium (4.69\%) (Fig. 2). There was a high inter-individual variability in the composition of the NPS and BAL microbiota across all the individuals (Fig. 2).

In NPS samples, we observed a predominance of Rathayibacter (24.41\% versus $0.07 \%$ in BAL samples), Mycoplasma (12.20\% versus $9.48 \%$ in BAL samples), and Corynebacterium (10.37\% versus $2.28 \%$ in in BAL samples).
While in BAL samples, we observed a predominance of Bibersteinia (14.41\% versus 0\% in NPS samples), Prevotella (11.69\% versus $0.28 \%$ in NPS samples) and Clostridium (6.85\% versus $2.53 \%$ in NPS samples). All other unassigned and classified OTUs belonged to genera comprising less than $1 \%$ of the total abundance represented as others/unassigned (Fig. 2).

Two bacterial phyla (Proteobacteria and Actinobacteria) showed a significant difference in relative abundance between NPS and BAL ( $P=0.041$ and 0.003 respectively). At the genus level, there were also statistical differences in abundances between these locations. For instance; Rathayibacter and Flavisolibacter Micrococcus, Agrococcus and Cellulomonas were more abundant in the
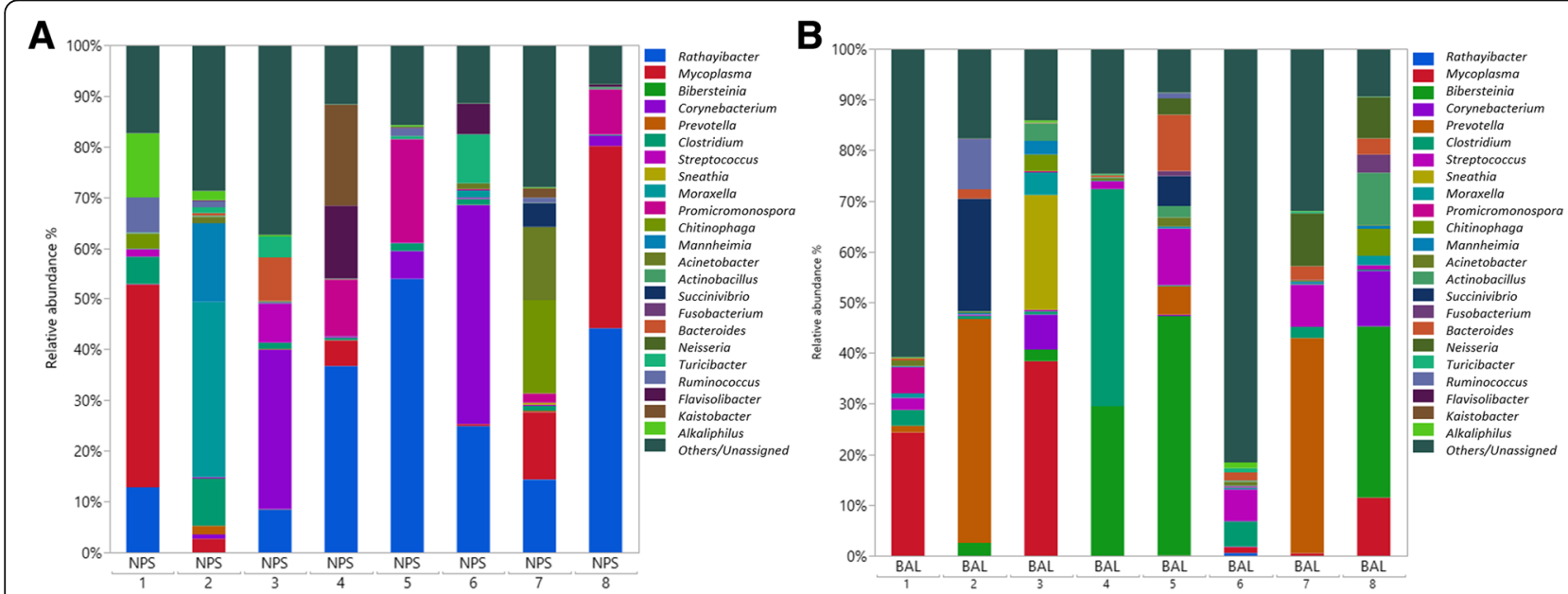

Fig. 2 Relative abundance of bacterial 16S rRNA gene sequences at the genus level observed in the NPS a and BAL $\mathbf{b}$ samples from 8 healthy feedlot calves. Only those bacterial genera that averaged more than 1\% of the relative abundance across all samples when sequencing V3-V4 hypervariable regions are displayed. All other unassigned and classified OTUs belonged to genera comprising less than $1 \%$ of the total abundance represented as others/Unassigned 
NPS $(P=0.004,0.028,0.040,0.009$ and 0.002 respectively) while Bibersteinia, Streptococcus and Bacteroides were more abundant in the BAL samples $(P=0.012,0.042$ and 0.023 respectively). Interestingly, there were no significant differences in the relative abundance of the organisms commonly associated with BRD (Mycoplasma, Mannheimia and Pasteurella,) between the NPS and BAL samples ( $P=0.512,0.071$ and 0.061 respectively).

LEfSe revealed that the NPS indicator OTUs were related to the bacterial genera Brachybacterium, Rathayibacter, Micrococcus and Flavisolibacter. Furthermore, the BAL indicator OTUs were related to the genera Leucobacter, Bacteroides, Streptococcus, Bibersteinia and Pasteurella (Fig. 3). The OTUs with the highest LDA (LDA log score threshold $\geq 2$ ) from each group are depicted in (Fig. 4). The relative abundance of the selected microbial taxa that displayed significant differences between NPS and BAL are shown in (Additional file 3: Fig. S1).

\section{Relationship between upper and lower airway microbial community structure.}

While there were both common and unique bacterial taxa present in both the NPS and BAL samples, the correlation analysis revealed strong associations between some of the most prevalent bacterial genera in both NPS (Additional file 4: Table S3 and Table S4) and BAL samples (Additional file 5: Table S5 and Table S6) at the population level, compatible with the concept of community structure.
There were highly significant correlations in the presence and abundance of some taxa (Mycoplasma, Sneathia, Moraxella, Mannheimia, Succinivibrio, Ruminococcus and Flavisolibacter) between the nasopharyngeal and lower respiratory samples (Table 1). In addition, the multiple linear forward regression analysis revealed that the presence of certain nasopharyngeal taxa was significantly associated with the presence of specific brochoalveolar taxa. For instance the presence of Streptococcus in NPS samples, was strongly predictive of the presence of Moraxella and Mycoplasma in BAL. Moreover, nasopharyngeal Bacteroides, Promicromonospora and Mycoplasma were strongly associated with the presence of brochoalveolar Mannheimia, Bibersteinia and Mycoplasma respectively (Table 2).

\section{Overall variation in airway microbial community structure and diversity}

The alpha diversity of the NPS and BAL communities in clinically healthy calves, was assessed using several indices (Table 3 and Additional file 6: Fig. S2). None of the alpha diversity indices differed significantly between NPS and BAL samples $(P>0.05)$.

The microbial community structure (beta diversity) of the NPS and BAL samples were significantly different from one another (ANOSIM $R$-value $=0.448, P=0.012$, weighted Unifrac and $R$-value $=0.386, P=0.022$, unweighted Unifrac), indicating a clear distinction between the NPS and BAL communities (Fig. 5).

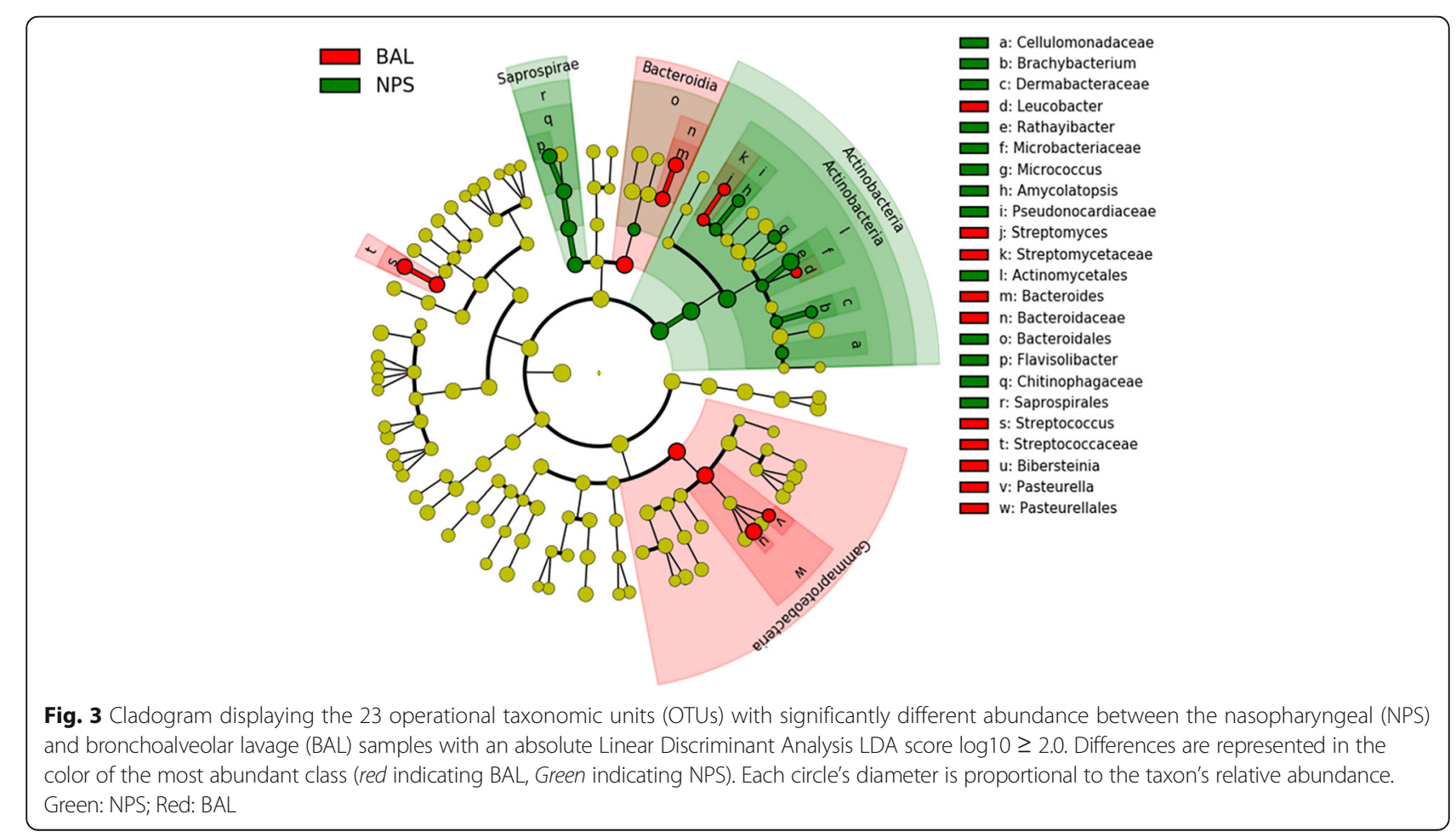




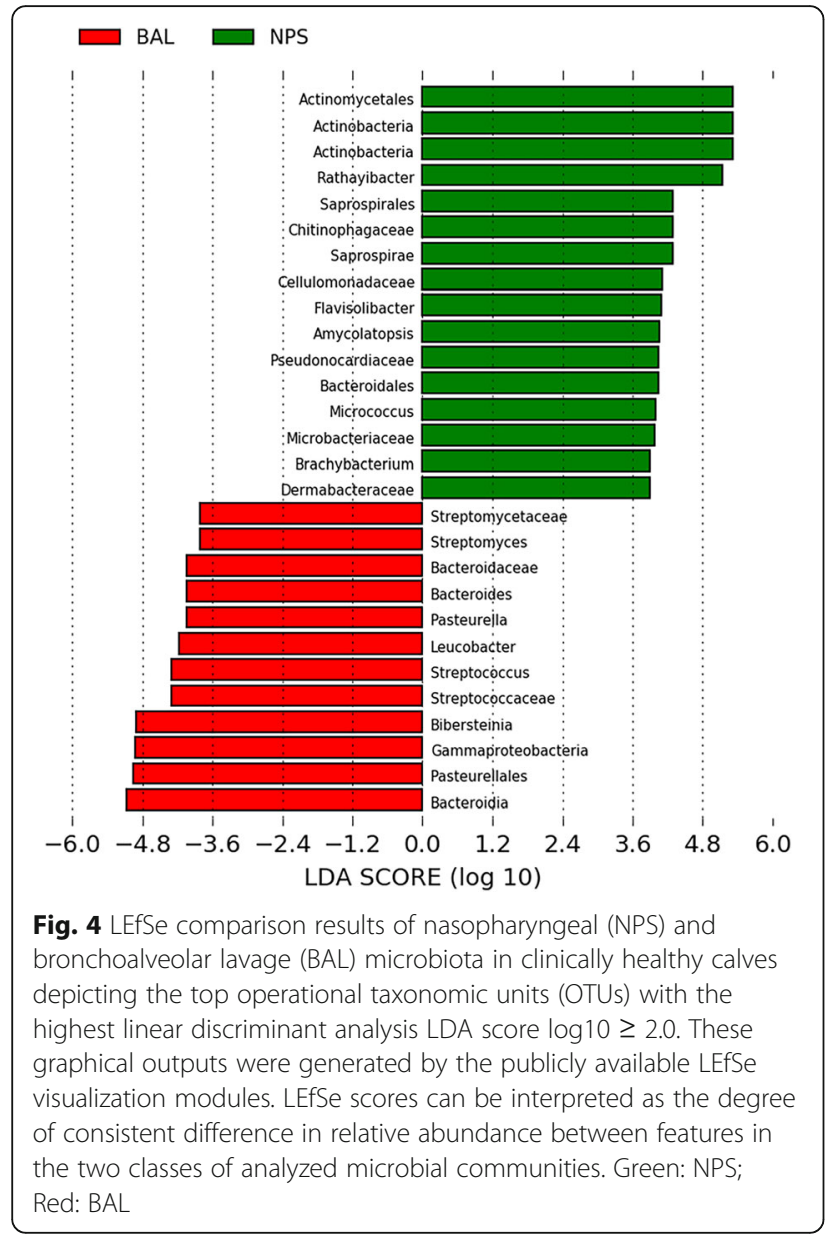
across all samples) between the nasopharyngeal (NPS) and bronchoalveolar lavage (BAL) samples in clinically healthy feedlot calves NP/BAL bacterial genera Rathayibacter Mycoplasma Bibersteinia Sneathia Moraxella Mannheimia Succinivibrio Ruminococcus Flavisolibacter Rathayibacter $0.913^{* *}$

Corynebacterium $0.777^{*}$

Prevotella

Clostridium

Streptococcus

Moraxella

Promicromonospora

Mannheimia

Fusobacterium

Bacteroides

Turicibacter

P value $<0.05{ }^{* *} P$ value $<0.01$

The table shows the significant Pearson correlation coefficient between locations. Statistical significance is indicated with asterisks
The core microbiota, defined as those OTUs found in all samples and identified at the genus level, of the respiratory tract was determined across all the NPS and BAL samples. In this population of animals, the core microbiota was composed of organisms from the Mycoplasma, Clostridium, Streptococcus, Moraxella and Alkaliphilus taxa.

\section{Discussion}

In this study we describe the first use of high throughput sequencing in comparing the upper and lower respiratory microbiota of feedlot cattle. These findings are important because of the central role played by the upper respiratory microbial communities in defense against potential pathogen access and colonization of the lung and lower airways $[9,11]$ and because the terminal airways and adjoining alveoli are the primary site of pathogen colonization and pathology in respiratory infections.

The calves in this study all originated from the same cow-calf enterprise, and had been exposed to similar management and environmental challenges, and so the pattern of nasopharyngeal microbial communities was anticipated to be relatively consistent across individuals. Despite the uniform management conditions, there was a high inter-individual variability in the composition of the NPS and BAL microbiota at both the phylum (Fig. 1) and genus level (Fig. 2). This is compatible with broader studies that show the multifactorial determinants (genetic, epigenetic, environmental, age, sex and dietary) underlying the establishment of the mucosal microbiota [31]. While the nutritional and environmental exposures would have been similar between these animals in this study, there would have been a significant variability in genetic and epigenetic influences across the population, which could have accounted for the observed differences. 
Table 2 Multiple linear forward regression analysis showing inter-relationship between the specific nasopharyngeal (NPS) and bronchoalveolar (BAL) taxa in clinically healthy feedlot calves

\begin{tabular}{|c|c|c|c|c|c|c|c|c|c|c|c|c|}
\hline \multirow[t]{2}{*}{ NPS/BAL bacterial genera } & \multicolumn{3}{|c|}{ Bibersteinia (BAL) } & \multicolumn{3}{|c|}{ Mycoplasma (BAL) } & \multicolumn{3}{|c|}{ Moraxella (BAL) } & \multicolumn{3}{|c|}{ Mannheimia (BAL) } \\
\hline & R square & $\begin{array}{l}\text { SE of the } \\
\text { estimate }\end{array}$ & $P$ value & R Square & $\begin{array}{l}\text { SE of the } \\
\text { estimate }\end{array}$ & $P$ value & R square & $\begin{array}{l}\text { SE of the } \\
\text { estimate }\end{array}$ & $P$ value & R square & $\begin{array}{l}\text { SE of the } \\
\text { estimate }\end{array}$ & $P$ value \\
\hline Mycoplasma (NPS) & & & & 0.977 & 0.075 & 0.001 & & & & & & \\
\hline Streptococcus (NPS) & & & & 0.769 & 0.025 & 0.004 & 0.842 & 0.006 & 0.001 & & & \\
\hline Promicromonospora (NPS) & 0.913 & 0.054 & 0.0001 & & & & & & & & & \\
\hline Actinobacillus (NPS) & 0.981 & 0.031 & 0.015 & & & & & & & & & \\
\hline Bacteroides (NPS) & & & & & & & & & & 0.950 & 0.002 & 0.0001 \\
\hline
\end{tabular}

SE standard error

This analysis was focussed on the taxa commonly associated with bovine respiratory pathogens

In our feedlot population the most predominant phylum (Proteobacteria) and genera (Rathayibacter, Mycoplasma, Bibersteinia, Corynebacterium, Prevotella, and Clostridium) were found across all NPS and BAL samples, and contributed to a common core microbiota of Mycoplasma, Clostridium, Streptococcus, Moraxella and Alkaliphilus for both of these sites. While the composition of the nasopharyngeal communities had some overlap with those previously reported in cattle at entry or after 60 days in the feedlot [10] there were some striking differences in our animals, specifically the complete absence of Carnobacterium and Shewanella, and the relatively low abundance of Mannheimia.

The relatively high abundance of Rathayibacter and Corynebacterium genus in NPS samples in our study is probably related to the high abundance of these organisms in the feedlot environment [32,33], and the fact that the nasal cavity is the first respiratory compartment exposed to microbes from the air, feed and water [10]. Corynebacterium species are also widely recognized as a common commensal of the skin and mucosal surfaces [34].

The relatively high abundance of the Mycoplasma genus in all NPS and BAL samples in our study, supports the observations of other investigators that members of this taxa (e.g. Mycoplasma dispar and Mycoplasma bovirhinis) are common, non-pathogenic inhabitants, and essential components of the core microbiota of the respiratory tract airways in healthy cattle $[9,34]$. The high frequency of occurrence of Mycoplasma species in our study also highlights the advantage of using culture-

Table 3 Bacterial diversity indices (Chao1, PD whole tree and Observed species) measures for the nasopharyngeal (NPS) and bronchoalveolar lavage (BAL) samples in clinically healthy feedlot calves

\begin{tabular}{llll}
\hline Calves group & Chao1 index & PD whole tree & Observed species \\
\hline NPS & $32.07 \pm 9.35$ & $2.77 \pm 1.41$ & $29.65 \pm 8.32$ \\
BAL & $28.75 \pm 10.14$ & $2.52 \pm 1.03$ & $24.22 \pm 9.36$ \\
\hline
\end{tabular}

The data are presented as the mean \pm standard deviation. There was no statistically significant difference in different bacterial diversity indices between the upper and lower respiratory tract $P$ value $>0.05$ independent techniques for the identification of organisms with fastidious growth requirements [10,35].

While there was some overlap between the upper and lower respiratory tract microbial communities, there was strong evidence for a distinct bronchoalveolar microbiota. For instance, the Bibersteinia were identified in BAL samples but were absent from the nasopharynx. The Bibersteinia genus belongs to the family Pasteurellaceae. These organisms have been reported as commensals of the nasopharynx and tonsils of healthy domestic sheep [36] and have been implicated as potential pathogens causing pneumonia in big horn sheep [37]. Bibersteinia has also been identified in both healthy and diseased cattle [38], but its occurrence in bovid species is unpredictable [39]. Since this is the first time that Bibersteinia has been reported as a common occupant of the lower respiratory tract of feedlot calves, further studies are needed to help understand its potential role in host health and productivity.

A particularly interesting aspect of our study was the identification of strong correlations between the presence of several specific taxa in nasopharyngeal samples and those in the lower respiratory tract. While the number of animals in this study was relatively small, and from a single source, these correlations are compatible with the notion of a mutualistic inter-relationship between the microbial communities at these two biogeographical locations. It has been shown that the healthy human lung does not harbor consistent and distinct microbial communities, but instead possesses a variable microbiota that is highly correlated, and largely indistinguishable from that of the upper respiratory tract [4]. The situation is altered somewhat in smokers, where the lung microbiome differs from the nasopharynx, but resembles that of the mouth [16]. This raises interesting questions regarding the process and route of microbial migration or translocation to the lungs from the environment, upper respiratory or gastrointestinal tract. In our study, the microbial populations of the upper and lower respiratory tract were clearly different from one another, which provided assurance that the sampling technique was relatively effective in preventing significant cross contamination between the upper and 

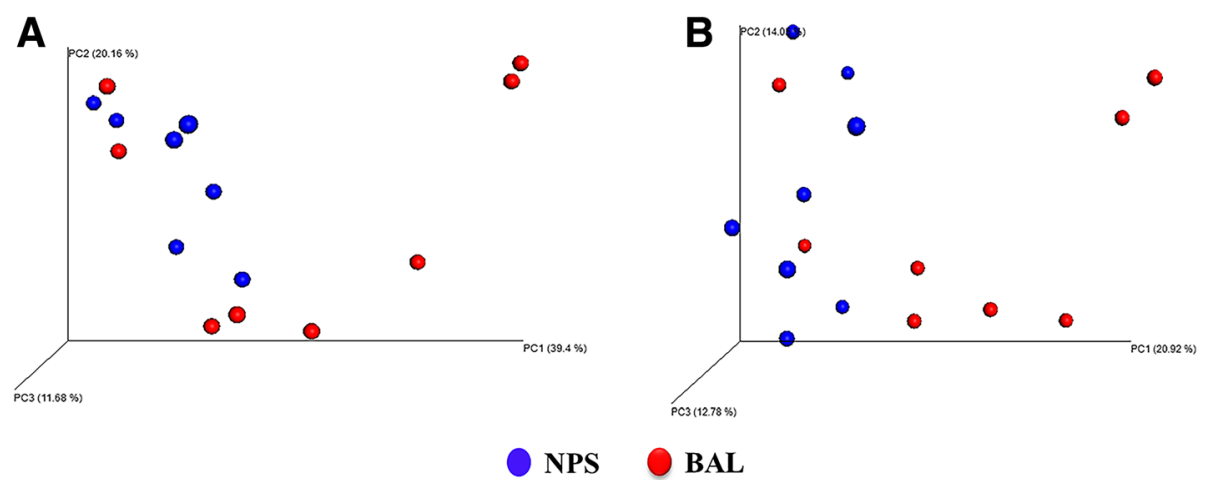

Fig. 5 Principal coordinate analysis (PCOA) of the weighted UniFrac distances $\mathbf{a}$ and un-weighted UniFrac distances $\mathbf{b}$ for the nasopharyngeal (NPS) and bronchoalveolar lavage (BAL) samples in clinically healthy feedlot calves. The percent variation explained by each principal coordinate is indicated on the axes. The individual data points from NPS (blue circle) and BAL (red circle) which represent total airway microbiota compositions of each calf are also depicted

lower respiratory airway. In addition, it was clear from the strong statistical correlations that, at each of the sites examined, the presence and abundance of one taxa was often closely related to that of another. In combination these findings support the idea that the microbes identified at each biogeographical location were not present in random haphazard patterns, but were inter-related and part of relatively structured populations. While this is the first report of the existence of a mutually interdependent, location-dependent microbiota community-structure along the respiratory tract of feedlot cattle, the concept aligns well with that currently accepted in human medicine $[16,40]$.

While the results of this study were compelling, and could open new avenues of investigation in bovine respiratory health, the three experimental limitations of our study somewhat hinder the applicability of these findings to the broader feedlot cattle population. Firstly, the analyses were performed on a relatively small number of animals. While this sample size was similar to that used in equivalent, published metagenomic studies in healthy and BRD-treated calves [2,10], data from a larger cohort of animals is required before definitive conclusions can be made regarding the specific taxa that constitute the lower respiratory microbiota in feedlot populations. Secondly, the potential for bronchoscopic contamination, or carryover, from the upper airway during passage of the external tube, could be viewed as potentially skewing the BAL microbial populations towards that present in the oropharynx or nasopharynx. The BAL technique was selected over a trans-tracheal approach, because we were interested in harvesting secretions from the smaller, more distal, airways as representative of the lower lung/airway microbiota. While we consider that the nasal approach circumvented the risk of significant oropharyngeal contamination, it was interesting to note that the NPS and BAL microbial populations in our study were clearly distinct from one another. It is possible that the high volume of fluid used for the BAL sampling procedure diluted the impact of any minor nasopharyngeal contamination on our results and conclusions. The last potential drawback in the study design was the absence of inclusion of negative controls in the sample analysis. It has been recently shown that a control sample, at the level of DNA extraction and processing, can mitigate the effects of contamination when examining low biomass environments [41]. At the time of this study, the inclusion of negative sequencing controls was not a widespread practice, and was not a part of equivalent studies $[7,9]$. In order to overcome the potential impact of low abundance contaminants on our results, a conservative approach to data interpretation was adopted. This approach meant that our analysis, interpretation, discussion, and conclusions were focused entirely on high abundance taxa, and on those populations that were significantly different between the two biogeographical locations.

As additional studies examining the respiratory microbiota are conducted in larger cohorts of animals from different environments, the notion of a relationship between the upper and lower respiratory tract microbiota could be a catalyst for a new trajectory of investigation in to bovine respiratory health. This is particularly exciting in view if the importance of BRD in the health, welfare and productivity of the cattle industry, and with regard to the potential for developing strategies that could help reduce the use of antimicrobials in this important branch of livestock-based food production.

\section{Conclusion}

In conclusion, this is the first study to describe the relationship between NPS and BAL microbiota in healthy feedlot cattle. In this cohort of cattle, the respiratory tract microbiota was dominated by Proteobacteria and Actinobacteria. The significant differences in the microbial community structure of the NPS and BAL samples 
indicated that a clear distinction exists between the microbiota at these sites. However, the strong correlations between the presence of several specific taxa in nasopharyngeal samples and those in the lower respiratory tract supports the notion of the existence of a mutualistic interrelationship between these biogeographically disparate microbial communities. Future studies, in large cohorts of animals, are needed to determine the role and clinical importance of the relationships of respiratory tract microbial communities with health, productivity, and susceptibility to the development of respiratory disease, in growing cattle.

\section{Additional files}

Additional file 1: Table S1. OTUs found in all NPS and BAL samples from 8 healthy feedlot calves. (XLSX $28 \mathrm{~kb}$ )

Additional file 2: Table S2. Relative abundance of the most common bacterial taxa at genus level for the NPS and BAL samples in clinically healthy feedlot calves. For each group the mean relative of predominant bacterial genera is reported. We determined statistically significant differences in relative abundance between groups (NPS and BAL) using nonparametric Wilcoxon tests.; $P$-values were calculated when statistically significant $(P<0.05)$. NS: non-significant $P$-value. (DOCX $17 \mathrm{~kb}$ )

Additional file 3: Fig. S1. Selected microbial taxa displaying significant differences in relative abundance between NPS and BAL. X-axis represent the relative abundance and $Y$-axis represent the individual samples. Straight line represents the mean abundance value of the group and the Dotted Line represents the median of the group. (DOCX $776 \mathrm{~kb}$ )

Additional file 4: Table S3. Correlation between the most prevalent bacterial genera in the nasopharyngeal swab samples. Table S4. Multiple linear forward regression analysis showing inter-relationship between the specific nasopharyngeal taxa in clinically healthy feedlot calves. (DOCX $15 \mathrm{~kb}$ )

Additional file 5: Table S5. Correlation between the most prevalent bacterial genera in the bronchoalveolar lavage samples. Table S6. Multiple linear forward regression analysis showing inter-relationship between the specific bronchoalveolar taxa in clinically healthy feedlot calves. (DOCX $16 \mathrm{~kb}$ )

Additional file 6: Fig. S2. Rarefaction curves of 165 rRNA gene sequences obtained from nasopharyngeal samples (NPS) and bronchoalveolar lavage samples (BAL) form clinically healthy calves. The graphical lines represent the mean and error bars represent standard deviations. The analysis was performed on a randomly selected subset of 2400 sequences per sample. (DOCX $194 \mathrm{~kb}$ )

\section{Abbreviations}

BAL: Broncho alveolar lavage; BRD: Bovine respiratory disease; DNA: Deoxyribonucleic acid; LDA: Linear discriminant analysis; LEfSe: Linear discriminant analysis effective size; NPS: Nasopharyngeal swabs; OTU: Operationa taxonomic unit; PCoA: Principle coordinate analysis; QIIME: Quantitative Insights Into Microbial Ecology; rRNA: Ribosomal ribo nucleic acid

\section{Acknowledgments}

We gratefully acknowledge the crew of DNA Services lab at the W. M. Keck Center for Comparative and Functional Genomics (University of Illinois at Urbana-Champaign, Urbana, IL) for performing the sequencing analysis.

\section{Funding}

The work was done at Department of Veterinary Clinical Medicine, University of Illinois at Urbana-Champaign and was funded through a joint supervision grant (JS 2986) from the Mission Sector, Ministry of Higher Education, Egypt.

\section{Availability of data and materials}

Fastq data obtained in the current study were uploaded to the sequence read archive (SRA) on National Center for Biotechnology Information (NCBI) website (http://www.ncbi.nlm.nih.gov/sra) to make the files available for a public database with bio-project accession number PRJNA323521.

\section{Author's contributions}

$J L$ and $B A$ designed the experiment. MZ, EG performed the experiment. MZ and MdG performed bioinformatics and statistical analysis. MZ, EG, MG, YA, and $J \mathrm{~L}$ wrote the manuscript. BA revised and edited the final version of the manuscript. All authors approved the manuscript submission.

\section{Competing interests}

The authors declare no competing interests.

\section{Consent for publication}

Not applicable.

\section{Ethics approval and consent to participate}

The use of animals for this study was approved by the University of Illinois Institutional Animal Care and Use Committee (IACUC Protocol: \#15064) and all of the experimental protocols were performed in accordance with relevant guidelines and regulations set by IACUC.

\section{Publisher's Note}

Springer Nature remains neutral with regard to jurisdictional claims in published maps and institutional affiliations.

\section{Author details}

${ }^{1}$ Integrated Food Animal Management Systems, Department of Veterinary Clinical Medicine, College of Veterinary Medicine, University of Illinois at Urbana-Champaign, 241 LAC, 1008 W Hazelwood Dr, Urbana, IL 61802, USA. ${ }^{2}$ Department of Animal Sciences and Division of Nutritional Sciences, University of Illinois at Urbana-Champaign, Urbana, USA. ${ }^{3}$ Department of Animal Medicine, College of Veterinary Medicine, Benha University, Benha, Egypt.

Received: 3 April 2017 Accepted: 2 June 2017

Published online: 23 June 2017

\section{References}

1. Edwards TA. Control methods for bovine respiratory disease for feedlot cattle. Vet. Clin. North am. - food Anim. Pract. 2010:26:273-84.

2. Portis E, Lindeman C, Johansen L, Stoltman G. A ten-year (2000-2009) study of antimicrobial susceptibility of bacteria that cause bovine respiratory disease complex-Mannheimia haemolytica, Pasteurella multocida, and Histophilus somni-in the United States and Canada. J Vet Diagn Investig. 2012;24:932-44

3. Apley M, Buczinski S, Forté G, Francoz D, Bélanger A-M, Burciaga-Robles LO, et al. The clinical syndrome of BRD: what it is and what it is not. Anim Health Res Rev. 2014;15:135-7.

4. Charlson ES, Bittinger K, Haas AR, Fitzgerald AS, Frank I, Yadav A, et al. Topographical continuity of bacterial populations in the healthy human respiratory tract. Am J Respir Crit Care Med. 2011;184:957-63.

5. Holman DB, McAllister TA, Topp E, Wright ADG, Alexander TW. The nasopharyngeal microbiota of feedlot cattle that develop bovine respiratory disease. Vet Microbiol. 2015;180:90-5.

6. Francoz D, Buczinski S, Bélanger AM, Forté G, Labrecque O, Tremblay D, et al. Respiratory pathogens in Qu??Bec dairy calves and their relationship with clinical status, lung consolidation, and average daily gain. J Vet Intern Med. 2015;29:381-7.

7. Weese JS, Slifierz M, Jalali M, Friendship R. Evaluation of the nasal microbiota in slaughter-age pigs and the impact on nasal methicillinresistant Staphylococcus aureus (MRSA) carriage. BMC Vet Res. 2014;10:69.

8. Durso LM, Harhay GP, Smith TPL, Bono JL, DeSantis TZ, Harhay DM, et al. Animal-to-animal variation in fecal microbial diversity among beef cattle. Appl Environ Microbiol. 2010;76:4858-62.

9. Li RW, Connor EE, Li C, Baldwin Vi RL, Sparks ME. Characterization of the rumen microbiota of pre-ruminant calves using metagenomic tools. Environ Microbiol. 2012;14:129-39. 
10. Holman DB, Timsit E, Alexander TW, Checkley SL, Campbell JR, Chirino-Trejo $\mathrm{M}$, et al. The nasopharyngeal microbiota of feedlot cattle. Sci. Rep. Nat Publ Group. 2015;5:15557

11. Slifierz MJ, Friendship RM, Weese JS. Longitudinal study of the early-life fecal and nasal microbiotasof the domestic pig. Bmc Microbiol. BMC Microbiol; 2015;15:12.

12. Timsit E, Workentine M, Schryvers AB, Holman DB, van der Meer F, Alexander TW. Evolution of the nasopharyngeal microbiota of beef cattle from weaning to 40 days after arrival at a feedlot. Vet Microbiol. 2016;187:75-81.

13. Gaeta NC, Lima SF, Teixeira AG, Ganda EK, Oikonomou G, Gregory L, et al. Deciphering upper respiratory tract microbiota complexity in healthy calves and calves that develop respiratory disease using shotgun metagenomics. J Dairy Sci. 2017;100:1445-58

14. Flanagan JL, Brodie EL, Weng L, Lynch SV, Garcia O, Brown R, et al. Loss of bacterial diversity during antibiotic treatment of intubated patients colonized with Pseudomonas Aeruginosa. J Clin Microbiol. 2007:45:1954-62.

15. Thorpe JE, Baughman RP, Frame PT, Wesseler TAS. Bronchoalveolar lavage for diagnosing acute bacterial pneumonia. Jour Infect Dis. 1987;155:855-61.

16. Erb-Downward JR, Thompson DL, Han MK, Freeman CM, McCloskey L Schmidt LA, et al. Analysis of the lung microbiome in the "healthy" smoker and in COPD. PLoS One. 2011:6

17. Charlson ES, Chen J, Custers-Allen R, Bittinger K, Li H, Sinha R, et al. Disordered microbial communities in the upper respiratory tract of cigarette smokers. PLoS One. 2010;5:1-10.

18. Beck JM, Young VB, Huffnagle GB. The microbiome of the lung. Transl. Res. Mosby. Inc. 2012;160:258-66.

19. Mang AV, Buczinski S, Booker CW, Timsit E. Evaluation of a computer-aided lung auscultation system for diagnosis of bovine respiratory disease in feedlot cattle. J Vet Intern Med. 2015;29:1112-6.

20. Godinho KS, Sarasola P, Renoult E, Tilt N, Keane S, Windsor GD, et al. Use of deep nasopharyngeal swabs as a predictive diagnostic method for natural respiratory infections in calves. Vet Rec. 2007;160:22-5.

21. Mitchell GB, Clark ME, Caswell JL. Alterations in the bovine bronchoalveolar lavage proteome induced by dexamethasone. Vet Immunol Immunopathol. 2007;118:283-93.

22. Welly RJ, Liu TW, Zidon TM, Rowles JL, Park YM, Smith TN, et al. Comparison of diet versus exercise on metabolic function and gut Microbiota in obese rats. Med Sci Sports Exerc. 2016:48:1688-98.

23. Caporaso JG, Kuczynski J, Stombaugh J, Bittinger K, Bushman FD, Costello EK, et al. Correspondence QIIME allows analysis of high- throughput community sequencing data intensity normalization improves color calling in SOLiD sequencing. Nat. Publ. Gr. Nat Publ Group. 2010;7:335-6.

24. Bokulich NA, Subramanian S, Faith JJ, Gevers D, Gordon Jl, Knight R, et al. Quality-filtering vastly improves diversity estimates from Illumina amplicon sequencing. Nat Methods. 2013;10:57-9.

25. Edgar RC, Haas BJ, Clemente JC, Quince C, Knight R. UCHIME improves sensitivity and speed of chimera detection. Bioinformatics [internet]. Oxford University Press. 2011;27:2194-200.

26. Edgar RC. Search and clustering orders of magnitude faster than BLAST. Bioinformatics. 2010;26:2460-1.

27. McDonald D, Price MN, Goodrich J, Nawrocki EP, DeSantis TZ, Probst A, et al. An improved Greengenes taxonomy with explicit ranks for ecological and evolutionary analyses of bacteria and archaea. ISME J. 2012;6:610-8.

28. Lozupone C, Knight R. UniFrac : a new phylogenetic method for comparing microbial communities UniFrac : a new phylogenetic method for comparing microbial communities. Appl Environ Microbiol. 2005;71:8228-35.

29. Vázquez-Baeza Y, Pirrung M, Gonzalez A, Knight R. EMPeror: a tool for visualizing high-throughput microbial community data. Gigascience. 2013;2:16.

30. Segata N, Izard J, Waldron L, Gevers D, Miropolsky L, Garrett WS, et al. Metagenomic biomarker discovery and explanation. Genome Biol. BioMed Central; $2011 ; 12:$ R60.

31. Spor A, Koren O, Ley R. Unravelling the effects of the environment and host genotype on the gut microbiome. Nat Rev. 2011;9:279-90.

32. Zgurskaya HI, Park J, Evtushenko LI, Akimov VN, Kalakoutskii LV. Rathayibacter gen. Nov., including the species Rathayibacter rathayi comb. nov., Rathayibacter tritici comb. nov., Rathayibacter iranicus comb. nov., and six strains from annual grasses. Int. J Syst Bacteriol. 1993;43:143-9.

33. Rintala H, Pitkaranta M, Toivola M, Paulin L, Nevalainen A. Diversity and seasonal dynamics of bacterial community in indoor environment. BMC Microbiol. 2008:8:56
34. Gao Z, Tseng CH, Pei Z, Blaser MJ. Molecular analysis of human forearm superficial skin bacterial biota. Proc Natl Acad Sci U S A. 2007:104:2927-32.

35. Maunsell FP, Donovan GA. Mycoplasma bovis infections in young calves. Vet. Clin. North am. - food Anim. Pract. 2009:25:139-77.

36. Tomassini L, Gonzales B, Weiser GC, Sischo W. An ecologic study comparing distribution of Pasteurella trehalosi and Mannheimia haemolytica between sierra Nevada bighorn sheep, White Mountain bighorn sheep, and domestic sheep. J Wildl Dis. 2009;45:930-40.

37. Dassanayake RP, Call DR, Sawant AA, Carol Casavant N, Weiser GC, Knowles $D P$, et al. Bibersteinia trehalosi inhibits the growth of mannheimia haemolytica by a proximity-dependent mechanism. Appl Environ Microbiol. 2010;76:1008-13.

38. Hanthorn CJ, Dewell RD, Cooper VL, Frana TS, Plummer PJ, Wang C, et al. Randomized clinical trial to evaluate the pathogenicity of Bibersteinia trehalosi in respiratory disease among calves. BMC Vet Res. 2014:10:8.

39. Panciera RJ, Confer AW. Pathogenesis and pathology of bovine pneumonia. Vet. Clin. North am. - food Anim. Pract. 2010;26:191-214.

40. Sze MA, Dimitriu PA, Hayashi S, Elliott WM, McDonough JE, Gosselink JV, et al. The lung tissue microbiome in chronic obstructive pulmonary disease. Am. J. Respir. Crit. Care Med. 2012;185:1073-80.

41. Salter SJ, Cox MJ, Turek EM, Calus ST, Cookson WO, Moffatt MF, et al. Reagent and laboratory contamination can critically impact sequence-based microbiome analyses. BMC Biol. 2014;12:87.

\section{Submit your next manuscript to BioMed Central and we will help you at every step:}

- We accept pre-submission inquiries

- Our selector tool helps you to find the most relevant journal

- We provide round the clock customer support

- Convenient online submission

- Thorough peer review

- Inclusion in PubMed and all major indexing services

- Maximum visibility for your research

Submit your manuscript at www.biomedcentral.com/submit 